\title{
L'université sans la nation : discours scolastiques
}

\section{Blaise Dufal}

\section{OpenEdition}

\section{Journals}

Édition électronique

URL : http://journals.openedition.org/ifha/8047

DOI : $10.4000 /$ ifha. 8047

ISSN : 2198-8943

\section{Éditeur}

IFRA - Institut franco-allemand (sciences historiques et sociales)

\section{Édition imprimée}

Date de publication : 31 décembre 2014

ISSN : 2190-0078

\section{Référence électronique}

Blaise Dufal, «L'université sans la nation : discours scolastiques », Revue de l'IFHA [En ligne], 6 | 2014 mis en ligne le 31 décembre 2014, consulté le 19 avril 2019. URL : http://journals.openedition.org/ ifha/8047 ; DOI : 10.4000/ifha.8047

Ce document a été généré automatiquement le 19 avril 2019

(CIFHA 


\title{
L'université sans la nation : discours scolastiques
}

\author{
Blaise Dufal
}

\section{La nation, mère du Moyen Âge français}

Pour Jules Michelet, «L'Angleterre est un empire, l'Allemagne un pays, la France une personne $»^{1}$. L'utilisation de la notion de personne, par l'un des historiens français inventeurs du Moyen $\hat{A} g e^{2}$, met en avant l'importance de la métaphore chrétienne de l'Incarnation dans la conception moderne de la nation. L'emploi de ce terme souligne également une nuance signifiante : la nation française n'est pas un individu pour Jules Michelet, c'est une personne ${ }^{3}$, distinction qui fait écho aux catégories scolastiques médiévales ${ }^{4}$. Cette distinction recouvre une série d'amalgames entre l'État, le peuple, la population et le territoire, et cherche à résoudre les tensions et les inadéquations à travers un processus de personnification visant à créer de l'unité5. Cependant, dans l'ensemble de son œuvre, Jules Michelet ne fait pas l'histoire de la nation, il fait l'histoire du peuple ${ }^{6}$, évacuant le plus possible la question de l'origine pour tenter d'échapper au récit continu de la conquête de la liberté dans le cadre de structures institutionnelles depuis l'essor communal du XIIe siècle. Il s'agit alors de s'opposer à une historiographie française bourgeoise et libérale marquée par Augustin Thierry qui fait du Moyen Âge dit « central » la matrice historique de la nation moderne française ${ }^{7}$. Également critique de cette vision de la nation, qui en retour construit une vision du Moyen Âge, Edgar Quinet remarque qu'en faisant de l'histoire de France une histoire sacrée, ces historiens appliquent « la méthode que les Pères de l'Église et les scolastiques ont si bien appliquée à l'histoire du peuple hébreu $\|^{8}$. Chez les historiens français du XIXe siècle, le discours sur la nation renvoie donc à la fois à un rapport personnel à l'obéissance politique mais aussi aux enjeux de la création du Moyen Âge comme champ disciplinaire et à la désignation de la scolastique comme modalité discursive de la fabrique de la nation.

Cet héritage reste présent dans l'identité disciplinaire de la médiévistique française au XXe siècle, servant de socle idéologique au positivisme français, ce qu'illustre l'un des 
éminents représentants de l'école méthodique, Charles Seignobos qui, en 1933, rédige l' Histoire sincère de la nation française. Dans cet essai, dont la vive critique faite par Lucien Febvre reste malheureusement toujours d'actualité, il affirme que «la plus ancienne civilisation indigène originale se crée au XIIe et au XIIIe siècle dans la région où la population a été renouvelée par les invasions franque et normande $»^{10}$. Ce moment fondateur se traduirait notamment par la création des universités, des collèges, des examens et des grades. Le Moyen Âge de cette historiographie française apparaît alors surtout comme le point crucial et nécessaire, pour l'existence d'un collectif, de la fixation des origines nationales. La nation n'est pas seulement un impensé, un inconscient, elle participe de la structure même du récit historique, elle sert de trame narrative qui permet de relier le contemporain au Moyen Âge et de légitimer l'existence de ce dernier ${ }^{11}$. Pourtant déjà Marc Bloch critique également ceux qui, comme Ernest Renan, ont la " hantise des origines ${ }^{12}$, réfutant scientifiquement et politiquement la pertinence des théories de l'auteur de Qu'est-ce qu'une nation? La nation est souvent décrite comme cette personne une et indivisible, évidente et insaisissable, à la fois réalisation historique et actrice de sa propre réalisation, forme qui donne sens à la matière collective, faisant écho bien souvent aux conceptions catholiques concernant le Saint Esprit et aux discours décrivant les relations trinitaires élaborées par la scolastique médiévale. La nation moderne est un incomparable ${ }^{13}$ qui a besoin des nations médiévales pour exister ${ }^{14}$ : mais les producteurs de savoir de la scolastique médiévale ont-ils besoin de la nation pour exister, c'est-à-dire pour penser le monde?

\section{Une identité très secondaire}

Une des caractéristiques des structures universitaires de l'Europe occidentale du XIIIe et $\mathrm{du}$ XIVe siècle est l'émergence de systèmes d'organisation distinguant et articulant des groupes sociaux dénommés nationes. Ces nations désignent des associations (societates) constituées d'enseignants et d'étudiants réunis selon leur région d'origine et/ou leur langue, chaque nation regroupant en général différents espaces de manières très diverses en fonction des universités. L'espace socio-économique et culturel de chaque producteur de savoir de la scolastique médiévale est a priori en partie structuré par son appartenance à une nation qui devrait se retrouver dans les systèmes d'identification culturelle des acteurs de ce champ ${ }^{15}$. Dans l'espace urbain qui caractérise la scolastique, les nationes fonctionnent à l'image des confréries, comme un dispositif de solidarité socioéconomique, une structure sociale d'accueil et de prise en charge des migrants, une modalité de représentation et de gestion des communautés d'étrangers : cette mobilité universitaire à l'échelle continentale pose en effet d'importants problèmes d'organisation matérielle et nécessite des structures d'encadrement. Le terme de nation décrit alors un système d'appartenance dans le cadre de la mobilité continentale des étudiants et des enseignants : c'est le large espace englobant d'où l'on vient, avant d'entrer dans le monde universitaire, et qui fait appartenir à tel ou tel groupe de solidarité dans le lieu où l'on est, où l'on travaille. Mais les nations universitaires restent des groupes assez flous, dont les spécificités linguistiques ou les limites territoriales sont fluctuantes; de plus, la division des membres de l'université en nations n'existe pas dans toutes les universités médiévales ${ }^{16}$. Les nations apparaissent alors plus comme des dispositifs d'ajustement liés à des situations locales, et non comme des traits nécessaires au phénomène universitaire. D'ailleurs, ce type d'organisation décline à la fin du XIVe siècle et au cours du XVe siècle ${ }^{17}$, 
au moment même où dans l'Europe occidentale des entreprises idéologiques sont menées par différents pouvoirs laïques pour construire des identités nationales spécifiques et exclusives. Les universités sont alors souvent mobilisées pour des objectifs politiques; et leur recrutement se fait de plus en plus au niveau local, un local qui se veut désormais défini comme national, en tant que territorial, entrainant la perte d'une certaine forme de continentalité du système universitaire ${ }^{18}$.

L'étude des autorités intellectuelles utilisées, citées et convoquées dans les écrits des scolastiques, permet de mettre en avant des catégories endogènes par lesquelles les acteurs définissent leurs identités, qu'elles soient religieuses, disciplinaires ou spatiales. Ces représentations dessinent leur statut social, leur système d'appartenance et les délimitations des différentes communautés qui constituent le champ de la scolastique. La plupart des autorités intellectuelles sont désignées dans les textes spéculatifs par leur nom et/ou leur prénom, et aussi souvent par leur surnom. Le nom renvoie très souvent à l'origine géographique du penseur, sans pour autant être articulé aux conceptions des nations des différentes universités. De nombreux producteurs de savoir de la scolastique sont désignés par un patronyme qui évoque leur lieu de naissance ou d'entrée dans un couvent, sans impliquer une appartenance collective ou un sentiment communautaire particulier. Le patronyme peut désigner un territoire vaste ou renvoyer à une localité très précise, mais cela ne relève pas d'une articulation entre l'individu et un collectif, qui permettrait d'ajouter une dimension supplémentaire à l'individu ainsi désigné et de lui conférer certaines caractéristiques psycho-culturelles liées à un groupe. Cet usage patronymique des localisations originelles est une pratique non spécifique aux scolastiques dans la société médiévale de l'Europe occidentale : ce qui est spécifique à la scolastique, c'est sans doute plutôt qu'aucune conséquence n'est dérivée de cette désignation territoriale. Les penseurs et les autorités ne sont pas situés dans leurs discours par rapport aux nations. Les nations universitaires des XIIIe-XIVe siècles n'apparaissent pas comme des formes d'identification des producteurs de savoir: les scolastiques ne se pensent pas au travers des nationes qui structurent pourtant en partie les universités médiévales.

Si l'université fut un des lieux majeurs de la distinction officielle entre nations, elle n'a pas pour autant produit de classification de ses autorités intellectuelles en fonction de leurs origines. Les producteurs de savoir de la scolastique ne se pensent pas les uns les autres en fonction d'une appartenance ethnico-politique. Ces désignations d'individus, devenues les emblèmes de contenus textuels, sont entièrement distinctes des catégories des nations de l'université médiévale. Si la scolastique voit apparaître certaines formes de patriotisme universitaire, celles-ci ne s'expriment pas selon des modalités nationales. Ces autorités intellectuelles obéissent plutôt à des systèmes de représentation qui sont spatialisées en fonction des lieux urbains d'exercice du savoir et de pratique professionnelle. Ces modes d'identification mettent en avant la dimension collective et cumulative de la production du savoir, l'importance du studium comme lieu de la pratique, et les filiations d'enseignants. Les théologiens traversent plusieurs communautés (ordre monastique, collège, université, discipline scolaire, structures politiques) mais ils appartiennent avant tout à l'élite de l'Église, à l'universitas qui est audessus des nations. Les discours scolastiques mentionnent les nations, mais l'anthropologie scolastique ne fait pas de la nation un concept identifiant et spécifiant les sociétés humaines. 


\section{De l'absent au multiple}

Les nations sont très peu présentes dans les développements à caractère politique produits par la scolastique médiévale des XIIIe et XIVe siècles. Ainsi, alors que Bernard de Clairvaux, formulant sa théorie des deux glaives à partir de l'exégèse d'un passage de l'Évangile de Luc $^{19}$, définit le pouvoir du pape par rapport aux différentes formes de regnum et de nationes, ce lieu textuel, pourtant très courant et au cœur des débats de l'époque, n'est pas l'occasion de développements importants sur les nations. Dans la longue polémique autour de cette citation et de ses interprétations, au cours des luttes politiques entre l'Église, l'Empire et les royautés, le terme de nation n'est quasiment pas discuté, développé ou travaillé20 dans les traditions exégétiques et scolastiques ${ }^{21}$. Cruciales dans l'historiographie de l'ecclésiologie chrétienne occidentale, les différentes utilisations de cette métaphore complexe ${ }^{22}$ soulignent, par l'absence, le peu d'intérêt qu'éprouvent les producteurs de savoir de la scolastique envers cette notion de nation. La nation n'apparaît alors pas comme une catégorie pertinente pour penser le politique. Les nations sont un donné de la société humaine que les scolastiques constatent éventuellement, sans l'interroger spécifiquement, sans l'inclure dans des typologies des formes d'appartenance sociale et identitaire, ni dans les descriptions des modalités de désignation collective et communautaire. Le dominicain Jean Quidort, théologien parisien à la fin du XIIIe siècle, qui soutient activement Philippe le Bel dans sa lutte contre le pouvoir pontifical de Boniface VIII, sort la métaphore des deux glaives de son enjeu politique ${ }^{23}$ et pose qu'ils représentent les deux Testaments. Le glaive divin évoque alors le verbe divin, et donc la prédication, mission fondamentale pour les théologiens scolastiques; le second glaive manifeste le martyr, autre forme possible d'action du chrétien dans le monde ${ }^{24}$. Pour les scolastiques, l'emploi de nationes renvoie donc très souvent au contexte de la prédication, désignant ainsi les destinataires de la parole cléricale.

Le terme de nation circonscrit, au pluriel, la diversité du monde, diversité irréductible des langues, conséquence du châtiment divin de la construction de la tour Babel, qui instaure la division et la confusion dans la société des hommes ${ }^{25}$. Les nationes sont très régulièrement convoquées dans les introductions ou les prologues des œuvres théologiques produites au XIIIe siècle et dans la première partie du XIVe siècle. Les expressions récurrentes d'omnes nationes et d'omnes orbis nationes font référence aux formules omni creaturae et omnes gentes de la parole évangélique, lorsque le Christ ressuscité enjoint aux apôtres de répandre son message dans le monde entier ${ }^{26}$. Le terme omnes semble d'ailleurs bien plus important que le terme nationes dans ces emplois scolastiques, insistant sur l'immensité de la création divine et sur la diversité des sociétés humaines. Il s'agit de souligner la volonté de saisir la totalité par le cumul des parties sans pour autant les dénombrer: les nationes sont les parties d'un tout qui permet de construire une globalité qui les dépasse. Les nations apparaissent, dans ces lieux textuels, comme une modalité importante pour dire la multiplicité des destinataires du message chrétien et de la prédication cléricale, fondamentale pour les théologiens des ordres mendiants, pour qui elle est non seulement une des activités majeures, mais aussi une forme centrale d'affirmation de leur identité sociale ${ }^{27}$. La plupart du temps, dans les discours scolastiques, le terme de nation est donc employé au pluriel, pour évoquer la multitude humaine et insister en retour sur l'étendue de la domination nécessaire de 
l'Église catholique pour en assurer l'unité. Les nationes, preuves de l'universalité de l'Église, participent alors d'une justification de l'entreprise hégémonique ecclésiale qui s'exprime notamment par le discours universaliste de la scolastique tant sur le plan intellectuel que culturel.

En-dehors des discours théologiques et politiques, les nationes apparaissent dans les marges de la scolastique, au sein des productions à caractère encyclopédique, historiographique et mythographique, pour désigner, entre autres termes, les peuples du passé, les sociétés antiques, les cultures païennes. Il s'agit dans ce cadre d'opérer une synthèse entre les référents bibliques et les propos des auteurs grecs et romains de l'Antiquité - ceux de Jules César notamment - pour décrire les sociétés d'avant le christianisme. De nombreux acteurs de la scolastique médiévale participent en effet à une importante production de savoirs sur les sociétés antiques païennes qui fonde et structure les connaissances mises en avant par les mouvements dits humanistes au cours du XIVe siècle ${ }^{28}$. À Oxford, au début du XIVe siècle, les dominicains Robert Holcot, Nicolas Trevet et Thomas Waleys et le franciscain John Ridevall composent des commentaires des auteurs de l'Antiquité païenne, dans lesquels ils construisent un savoir proprement consacré aux cultures antiques païennes. Ces fratri classici ${ }^{29}$, s'inscrivant dans les traditions encyclopédiques médiévales et les développant, traitent des nationes, c'est-àdire, dans ce cas, de la diversité des religions et des cultes, ainsi que de la multiplicité des croyances et des représentations du divin. Si le terme de nation est employé pour définir les entités socio-culturelles du passé dans une démarche anthropologique visant à mieux les décrire et les comprendre, le terme de populus apparaît néanmoins bien plus crucial que celui de nationes ou de gens. L'objet de l'interrogation de ces scolastiques est de comprendre les caractéristiques d'autres populations en se centrant sur la dimension religieuse, la multiplicité des nations n'impliquant pas une diversité religieuse, bien au contraire. Les nations ne sont donc pas spécifiquement utilisées pour penser l'altérité religieuse, pas plus que pour désigner l'étranger ou le peuple lointain ou proche. Dans le regard scolastique sur le passé de la société chrétienne, les nations sont présentes mais ne constituent pas une catégorie efficiente pour la description historico-anthropologique du monde.

\section{Mon nom est personne}

La nation n'apparaît pas comme une catégorie structurante pour la scolastique, ni en tant que modalité de distinction des acteurs de ce champ de production culturel, ni en tant que catégorie d'analyse du monde. Ce caractère périphérique et accessoire doit être compris par rapport à la construction par les institutions scolastiques d'un discours universaliste, fondement des savoirs chrétiens occidentaux. Les nations participent de la totalité universelle, un ensemble de parties équivalentes dont le nombre forme un tout qui les dépasse et que les scolastiques distinguent de la totalité intégrale, où chaque élément est nécessaire au tout et où la totalité cesse d'exister quand la partie manque. Intégrées dans une conception universaliste ecclésiale, au sens propre catholique, les nationes sont une composante mineure des formes de représentation du monde et du rapport de soi à l'autre dans la scolastique médiévale. Le caractère essentialiste et naturaliste de la conception moderne est en effet incompatible avec l'ontologie analogiste à l'œuvre dans les sociétés médiévales et avec les systèmes combinatoires propres à la scolastique. L'universalisme scolastique, contrairement à l'universalisme moderne 
occidental des Lumières, n'envisage pas la tension divergente entre le tout et les parties comme fondement de l'espace politique, bien au contraire. L'adéquation entre un territoire, une culture et une langue est profondément étrangère à ces clercs, pérégrinant à travers le continent, parlant une même langue et partageant une culture dont la catégorie du national ne fait pas partie. L'universalisme scolastique se construit par l'abstraction théologique qui convertit les données révélées et les mystères de la foi en une science ${ }^{30}$. Discours de l'unité et de la complexité du monde, sur le modèle de la Summa , ordonnancement logique distinguant chaque possible du réel et reliant ces possibles entre eux pour en faire un tout signifiant, la casuistique scolastique articule par l'abstraction l'aspiration à l'universalité ${ }^{31}$. Les nations sont alors des cas particuliers ${ }^{32}$ qui disent l'universalité du christianisme alors que la nation apparait comme une personne étrangère à l'individu de la scolastique.

La conception moderne et occidentale de la nation, telle qu'exprimée par Jules Michelet, correspondrait dans la scolastique médiévale à une fiction juridique ${ }^{33}$, création spéculative insistant sur plutôt sur les ressemblances que sur les différences entre certains humains. La nation moderne n'est pas un individu, au sens scolastique du terme ${ }^{34}$ , c'est une personne morale, une personne fictive, qui se vit et se pense selon les modalités de l'Incarnation. Cette nation évoque alors plus la conception de l'Église comme personne, dans sa monumentalité, qui prend forme à partir de la réforme grégorienne et des développements du droit canon ${ }^{35}$. La généalogie de la nation moderne doit alors être inscrite plutôt dans des usages modernes laïcisants des concepts de l'ecclésiologie catholique. Absolue et universelle comme l'Église catholique, la nation moderne est conçue comme corps collectif, disposant d'un statut socio-juridique, c'est-à-dire une personne, au sens scolastique. Cette persona, qui signifie en latin classique le masque de théâtre, désigne dans la théologie trinitaire et depuis Boèce, l'existence d'une substance en rapport avec l'essence divine. La personne, pour les scolastiques, est fondamentalement relation, relation à la transcendance. Définie au cours des débats du XIIIe siècle sur les puissances de l'âme, cette notion fonctionne comme une unité de substitution permettant d'articuler l'existence individuelle à l'histoire du salut collectif ${ }^{36}$. Dans cet espace relationnel, l'historien, s'il le voulait, pourrait trouver la trace d'un dispositif conceptuel rendant possible la notion moderne de la nation. En effet, la nation moderne fonctionne comme une transcendance qui articule entre eux des individus, en tant que dignités humaines distinctes, reliés à une fraction indivisible et universelle de l'espèce humaine. Mais la nation moderne ne peut exister tant que les producteurs de savoir de la scolastique pensent de "vagues individus», difficilement soumis aux pouvoirs humains et peu réductibles à leurs origines ou à leurs appartenances. Loin de l'illusion moderne de la nation fanion, les nations de la scolastique ne sont que des contingences historiques et non des armes politiques.

\section{NOTES}

1. Jules Michelet, Histoire de France, t. 2, Paris : Chamerot, 1861, livre III, p. 103. 
2. Jacques Le Goff, «Les Moyen Âge de Michelet », in : Pour un autre Moyen Âge, Paris : Gallimard, 1977, p. 19-45. Jules Michelet est d'ailleurs aussi considéré comme l'un des inventeurs de la Renaissance : Lucien Febvre, Michelet et la Renaissance, Paris : Flammarion, 1992 ; Simone BernardGriffith (dir.), Michelet entre naissance et Renaissance (1798-1998), Clermont-Ferrand/Paris : Presses universitaires Blaise Pascal, 2001.

3. «Cette prégnance des représentations de l'espace est caractéristique de la pensée historique de Michelet. Elle explique en particulier que la France lui soit apparue comme une personne. Michelet tenait beaucoup à cette métaphore, qu'il affirmait avoir inventée ( le premier je la vis [la France] comme une âme et une personne »). L'assimilation d'une nation à un individu était pourtant une opération d'écriture très banale à l'époque. On en trouverait de nombreux exemples, depuis Sismondi jusqu'à Henri Martin. Mais le cheminement qui conduisit Michelet à faire de la France non pas exactement un individu, mais une âme et une personne, était en effet original.» (Sylvain Venayre, Les Origines de la France. Quand les historiens racontaient la nation, Paris : Édition du Seuil, 2013, p. 90-91).

4. Pour Jules Michelet, les états généraux de 1302, sous le règne de Philippe le Bel, inaugurent «l'ère nationale de la France, son acte de naissance » (Jules Michelet, Histoire de France, t. 3, Paris, 1837, p. 55).

5. Si il y a une "identité nationale française ", elle se situe sans doute dans la confusion profonde entre toutes les notions agrégées autour du terme de nation, confusion masquée par la proclamation de l'évidence de l'existence de la nation.

6. Paul Viallaneix, La Voie royale. Essai sur l'idée de peuple dans l'œuvre de Michelet, Paris : Delagrave, 1959.

7. « Nous sommes ici à l'origine du monde social des Temps modernes » (Augustin Thierry, Essai sur l'histoire de la formation et des progrès du tiers état, Paris : Furne, 1853, p. 16).

8. « Nous mettons notre honneur à nous faire dès l'origine serfs d'autrui et à dater notre histoire du premier jour de notre esclavage "; "Appliquant à nos origines je ne sais quel mysticisme scolastique, il nous plaît que nos ancêtres soient d'abord asservis et extirpés pour nous donner ensuite le spectacle de leur lente et incertaine résurrection" (Edgar Quinet, "Philosophie de l'histoire ", Revue des deux mondes, janvier-février 1855, p. 929 et p. 933).

9. «Nation, écrivez-vous. Mais quoi, nation? De quand date le mot? Quels synonymes lui ont fait cortège ? Et comment les distinguait-on de lui ? Surtout, derrière ces mots, quelles réalités les Français ont-ils entassées successivement? Tant que vous n'avez pas dit - ou essayé de dire - cela, vous n'avez rien dit pour un historien. Vous avez seulement renforcé la tendance à prendre les mots du langage les plus clairs aujourd'hui pour les hommes aujourd'hui, comme autant de véhicules confortables et sûrs pour remonter le cours des siècles, bien assis, sans avoir besoin de changer de place, ou de voiture " (Lucien Febvre, « Entre l'histoire à thèse et l'histoire-manuel. Deux esquisses récentes d'Histoire de France : M. Benda, M. Seignobos ", Revue de synthèse, $\mathrm{t} . \mathrm{V}, \mathrm{n}$ -3, décembre 1933, p. 205-236, voir p. 212). Sur ce débat voir: Antoine Prost, "Seignobos revisité ", Vingtième Siècle, Revue d'histoire, $\mathrm{n}^{\circ}$ 43, 1994, p. 100-108.

10. Charles Seignobos, Histoire sincère de la nation française, Paris : Presses universitaires de France, 1969 , p. 340.

11. János M. Bak et alii (dir.), Gebrauch und Missbrauch des Mittelalters, 19.-21. Jahrhundert, München : W. Fink, 2009.

12. Marc Bloch, Apologie pour l'histoire ou le métier d'historien, Paris : Armand Colin, 1997, p. 53.

13. Marcel Détienne, Comparer l'incomparable, Paris : Édition du Seuil, 2000.

14. Patrick J. Geary, The Myth of Nations, Princeton :Princeton University Press, 2002.

15. Comme les facultés, les collèges et les universités, les nations ont leurs saints protecteurs et leurs fêtes.

16. Jacques Verger, «Les universités comme corporation », in : Les Universités au Moyen Âge, Paris : Presses Universitaires de France, 1973, p. 47-78. 
17. Le déclin du système des nations à la fin du Moyen Âge manifeste la transformation du recrutement universitaire; les anciennes nations perdent l'essentiel de leurs prérogatives et, dans les nouvelles universités, on n'en crée souvent pas (Pearl Kibre, The Nations in the Mediaeval Universities, Cambridge Mass. : Mediaeval Academy of America, 1948).

18. "Les universités perdent aussi, au cours de ces deux siècles, leur caractère international. La cause principale en est la fondation de nombreuses universités nouvelles dont le recrutement prend un aspect de plus en plus national ou même régional » (Jacques Le Goff, Les Intellectuels au Moyen Âge, Paris : Édition du Seuil, 1957, p. 156).

19. Luc, XXII, 35-38.

20. Jacques Leclerc, «L'argument des deux glaives (Luc XXII, 38) dans les controverses politiques du Moyen Âge ", Recherches de science religieuse, t. 21, 1931, p. 299-329; t. 22, 1932, p. 150-177 et p. 280-303.

21. Philippe Buc, L'Ambiguité du livre. Prince, pouvoir et peuple dans les commentaires de la Bible au Moyen Âge, Paris : Beauchesne, 1994.

22. Yves M. congar, L'Église de saint Augustin à l'époque moderne, Paris : Édition du Seuil, 1997, p. $142-145$.

23. Cette attitude peut faire écho à celle du franciscain Pierre de Jean Olivi, pour qui la réponse $\mathrm{du}$ Christ doit se comprendre ironiquement, le Christ se moquant de ceux qui lui suggèrent de prendre les armes pour se défendre. «La théorie des deux glaives ne s'imposait donc pas comme un donné scripturaire intangible» (Alain Boureau, La Religion de l'État, Paris : les Belles Lettres, 2006, p. 222).

24. Johannes Quidort von Paris, De regia potestate et papali : Fritz Bleienstein (dir.), Über königliche und päpstliche Gewalt, Stuttgart: E. Klett, 1969.

25. Benoît Grévin, Le Parchemin des cieux. Essai sur le Moyen Âge du langage, Paris : Édition du Seuil, 2012, p. 122-128.

26. Marc, 16-15: «Euntes in universum mundum praedicate Evangelium omni creaturae "; Matth. 28-19: « Euntes ergo docete omnes gentes baptizantes eos in nomine Patris et Filii et Spiritus Sancti».

27. Nicole Bériou, L'Avènement des maîtres de la parole. La prédication à Paris au XIIIe siècle, Paris : Institut d'Études Augustiniennes, 1998.

28. Nicholas Mann/Birger Munk Olsen (dir.), Medieval and Renaissance Scholarship, Leiden/New York : Brill, 1997 ; Leighton D. Reynolds (dir.), Texts and Transmission. A Survey of the Latin Classics, Oxford : Clarendon, 1983.

29. Beryl Smalley, English Friars and Antiquity in the Early Fourteenth Century, Oxford : B. Blackwell, 1960.

30. Marie-Dominique Chenu, La Théologie comme science au XIIIe siècle, Paris: Librairie philosophique J. Urin, 1957.

31. Yan Thomas, «L'extrême et l'ordinaire. Remarques sur le cas médiéval de la communauté disparue ", in: Jean-Claude Passeron, Jacques Revel (dir.), Penser par cas, Paris: Édition de l'EHESS, 2005, p. 45-73.

32. «Les penseurs scolastiques réduisaient donc le référent, le monde naturel soit à une métaphore instrumentale et fonctionnelle, soit à un cas précis, relevant de la connaissance des particuliers. L'investissement dans le particulier relevait soit d'un nécessaire complément à l'universalité, manifestée par l'abstraction, soit d'une réaction polémique contre celle-ci » (Alain Boureau, L'Empire du livre, Paris : les Belles Lettres, 2006, p. 199).

33. Yan Thomas, "L'empire de la fiction romaine et ses limites médiévales", Droits, revue française de théorie, de philosophie et de culture juridique, $\mathrm{n}^{\circ}$ 21, 1995, p. 17-63.

34. «La personne désignait une entité étrange, qui ne pouvait se réduire à une institution publique sans pouvoir se traiter comme individu " (Alain Boureau, De vagues individus. La condition humaine dans la pensée scolastique, Paris : Les Belles Lettres, 2008, p. 260). 
35. «Au nom des substances secondes l'Église est elle-même une persona et [...] la sociabilité des groupes dans le Moyen Âge occidental (Ecclesia, universitas, communitas, corpus, congregatio, collectio, collegium) met fondamentalement en rapport deux personnes - la personne singulière du fidèle, et celle, morale et fictive, de l'Église - comme si l'individu, irréductiblement seul face à Dieu, ne pouvait trouver son intelligibilité substantielle que dans la communion ecclésiale » (Dominique Iogna-Prat, "La question de l'individu à l'épreuve du Moyen Âge ", in: Brigitte M. Bedos-Rezak/Dominique Iogna-Prat (dir.), L'Individu au Moyen Âge. Individuation et individualisation avant la modernité, Paris : Aubier, 2005, p. 28 ; Dominique Iogna-Prat, La Maison Dieu : une histoire monumentale de l'Église au Moyen Âge, Paris : Édition du Seuil, 2006.

36. Alain Boureau, De vagues individus, op. cit.

\section{AUTEUR}

\section{BLAISE DUFAL}

EHESS, Paris (Groupement d'anthropologie scolastique) 